\title{
CONSTRUCCIÓN Y TRANSFORMACIONES DE LAS IDENTIDADES ACADÉMICAS DE ESTUDIANTES DOCTORALES MEDIANTE LA CITACIÓN
}

\author{
CONSTRUCTION AND TRANSFORMATIONS OF DOCTORAL STUDENTS' ACADEMIC IDENTITIES \\ THROUGH CITATIONS
}

CONSTRUCTION ET TRANSFORMATIONS DES IDENTITÉS ACADÉMIQUES DES DOCTORANTS À TRAVERS LA CITATION

\author{
Lina Calle-Arango \\ Maestra en Educación, Pontificia \\ Universidad Católica de Chile, \\ Santiago. \\ Candidata a doctora en Educación, \\ Pontificia Universidad Católica de \\ Chile, Santiago, Chile. \\ Icalle@uc.cl \\ https://orcid. \\ org/0000-0003-4362-3075
}

\section{Natalia Ávila Reyes}

Doctora en Educación, Universidad de California, Santa Bárbara, EE.UU.

Profesora asistente, Facultad de Educación, Pontificia Universidad Católica de Chile, Santiago.

naavila@uc.cl

https://orcid.

org/0000-0003-3528-6806

\section{Alejandra Meneses \\ Doctora en Lingüística, Pontificia \\ Universidad Católica de Valparaíso, \\ Valparaíso, Chile. \\ Profesora asociada, Facultad de Educación, Pontificia Universidad Católica de Chile, Santiago. \\ amenesea@uc.cl \\ https://orcid. \\ org/0000-0002-6563-5117}

\begin{abstract}
RESUMeN
Los desafíos y las tensiones identitarias de los doctorandos constituyen un tema de creciente relevancia. Una manera de estudiarlo es mediante la citación, pues para participar del mundo académico, el investigador en formación debe posicionarse en diálogo con otros autores y con los avances de su comunidad discursiva. Esta investigación utiliza el marco teórico de las literacidades académicas para analizar longitudinalmente, la relación entre la citación y la construcción de identidad académica de cuatro doctorandos en Educación de una universidad chilena altamente selectiva. Para esto se tomaron en cuenta los propósitos de citación en las introducciones de tres versiones de una revisión de literatura que estos escribieron. Dicho análisis se integró con sus testimonios, los cuales fueron recogidos por medio de entrevistas basadas en textos. Los resultados muestran cómo los doctorandos eligieron propósitos de citación correspondientes a hábitos, estilos o filiaciones disciplinarias, así como a la construcción de la imagen que deseaban proyectar como investigadores, la cual los sitúa a medio camino hacia una autopercepción como tales. Este contraste les permitió la construcción de una identidad académica híbrida o múltiple.
\end{abstract}

Palabras clave: citación; escritura académica; estudiantes doctorales; identidad académica; intertextualidad.

\section{Abstract}

Doctoral students' challenges and identity tensions are becoming an increasingly relevant topic. One way of studying this is through the analysis of citations, since, to participate in the academic world, doctoral students must enter into dialogue with other authors and with advances in their discourse communities. This research uses the theoretical framework of academic literacies to analyze, longitudinally, the relationship between the citations and academic identity building of

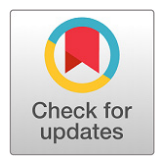

Recibido: 2020-11-24/ Aceptado: 2021-02-02 / Publicado: 2021-05-05

https://doi.org/10.17533/udea.ikala.v26n2a12 
four Education doctoral students enrolled in a highly selective Chilean university. To determine the relationship between citation and academic identitybuilding, the citation' purposes of the introductions of three versions of the literature reviews written by these students were analyzed. Such analisis was complemented with students testimonies, which were collected through text-based interviews. The results show how citations' purposes were chosen based on habit, style or disciplinary affiliations, as well as on the image they wanted to project as researchers, which places them halfway to a self-perception as such. This contrast allowed them to build a hybrid or multiple academic identity.

Keywords: citation; academic writing; doctoral students; academic identity; intertextuality.

\section{RÉSUMÉ}

Les défis et les tensions identitaires des doctorants constituent un sujet de plus en plus pertinent. Une façon de l'étudier est par la citation, car pour participer au monde académique, le chercheur en formation doit se positionner dans le dialogue avec d'autres auteurs et avec les avancées de sa communauté discursive. Cette recherche utilise le cadre théorique des littératies académiques pour analyser, dans une perspective longitudinale, la relation entre la citation et la construction de l'identité académique de quatre doctorants en éducation d'une université chilienne très sélective. Pour cela, les objectifs de la citation ont été pris en compte dans les introductions de trois versions d'une revue de littérature qu'ils ont rédigée et ces versions ont été intégrées à leurs témoignages, qui ont été recueillis par le biais d'entretiens axés sur les textes. Les résultats montrent comment les doctorants ont choisi des objectifs de citation en raison des habitudes, des styles ou des affiliations disciplinaires, ainsi que pour la construction de l'image qu'ils voulaient projeter en tant que chercheurs et qui les place à mi-chemin vers une perception de soi en tant que tels. Ce contraste leur a permis de construire une identité académique hybride ou multiple.

Mots-clés : citation : écriture académique ; doctorants ; identité académique ; intertextualité.

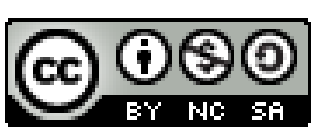




\section{Introducción}

Los estudios sobre la relación entre escritura e identidad han crecido fuertemente en los últimos años, en particular desde marcos sociolingüísticos y perspectivas como los Nuevos Estudios de Literacidad. Desde esta posición, se asume que las identidades influyen y son influidas por la participación en diversas prácticas escritas; en otras palabras, quién es y en qué aspira a convertirse un escritor intervienen en la elección de prácticas en la que se involucra, los propósitos que espera cumplir con ellas, el significado que construye a partir de los textos y los valores que asocia a esos textos y prácticas (Ivanič et al., 2009).

Por su parte, los estudios sobre el desarrollo de la identidad académica, especialmente en doctorandos e investigadores en etapas tempranas de su carrera, han surgido con fuerza durante las últimas décadas (véanse Giampapa, 2011; Leshem, 2020). En una revisión sistemática de literatura sobre identidad académica, Castelló et al. (2020) identificaron cuatro grandes grupos (transición entre identidades, equilibrio entre continuidad y cambio de identidad, desarrollo de la identidad personal a través del tiempo e identidad personal y estable), según la visión de la identidad subyacente en los estudios empíricos de las últimas dos décadas; los autores combinaron variables de identidades múltiples o únicas, con un carácter social o individual en su definición. Esta variabilidad en las posibilidades de combinación de estas dimensiones, encontrada por Castelló et al., confirma la complejidad del concepto de identidad. En efecto, la identidad académica involucra la negociación entre una identidad individual que se construye socialmente y otra que se asume en los roles institucionales y comunitarios; del mismo modo, un investigador en formación puede tener identidades previas, de otras disciplinas o profesiones (Hoang y Pretorius, 2019), que están constantemente en reformulación y diálogo con nuevas posibilidades de ser.

Todas las actividades en las que participan los individuos les permiten generar un sentido de identidad, una idea sobre con qué se identifican y a qué grupo pertenecen. En el contexto académico, esta construcción tiene lugar mediante el acto de investigar, que modela la forma en que los investigadores se perciben como miembros de su campo (Hoang y Pretorius, 2019). De allí que el estudio de la identidad académica converja tan poderosamente con la escritura, comprendiendo que gran parte de la actividad académica consiste precisamente en escribir (Tusting et al., 2019).

El desarrollo de la identidad académica mediante la escritura en estudiantes doctorales ha sido abordado en el entendido de que estos forjan sus nuevas identidades como investigadores en el ejercicio de la escritura académica (Castelló etal., 2013; Inoyue y McAlpine, 2019). Las investigaciones recientes sobre identidad desde perspectivas socioculturales han tendido a focalizarse en dinámicas de retroalimentación y supervisión, experiencias en cursos o grupos de escritura, y vivencias de estudiantes internacionales (Inoyue y McAlpine, 2019), mientras los estudios que involucran el uso de la intertextualidad académica como estrategia de construcción de una identidad del investigador novel son mucho más escasos.

Desde la retórica de la ciencia y el construccionismo social, se ha mostrado que las citas tienen un carácter estratégico y persuasivo: más que constituir un reporte estrictamente objetivo de fuentes utilizadas (Bornmann y Daniel, 2008), representan formas de argumentación y de alineamiento científico, social e incluso mercantil (Erikson y Erlandson, 2014). Al citar, el investigador construye un ethos, una credibilidad dada por las fuentes que se citan (Harris, 2005), mediante elecciones textuales que demuestran al lector quién se es, con quién(es) se afilia e identifica el escritor (y, por lo tanto, también de quién(es) se opone o diferencia), la comunidad a la que pertenece o quiere pertenecer (Ávila Reyes, 2018; Sapienza, 2007).

Entre los escasos estudios sobre citación como estrategia de construcción de la identidad del investigador posgradual están los de Fazel y Shi (2015); Harwood y Petrić (2012) y Qingrong y Liming (2019). 
Fazel y Shi (2015) indagan en los comportamientos de citación de seis estudiantes doctorales de ciencias sociales y humanidades, en una universidad canadiense, durante la escritura de sus propuestas de investigación. Valiéndose de entrevistas semiestructuradas, los autores plantean que para construir y reflejar su identidad académica en formación, los estudiantes optan por citar para demostrar conocimiento de las autoridades en su campo y apoyarse en estudios o investigadores anteriores; asimismo, recurren a estrategias como imitar a otros, disimular la poca familiarización con un tema o seguir las sugerencias de sus tutores.

Por su parte, Harwood y Petrić (2012) realizan entrevistas basadas en textos a dos estudiantes internacionales de una maestría en Administración de Empresas de una universidad británica, y las analizan bajo la metáfora de la actuación de Goffman (1959) para la construcción de una imagen frente al lector. Los resultados muestran que las decisiones de cantidad e inclusión de determinadas referencias constituyen estrategias para proyectar al lector imágenes de atención, conocimiento de temáticas, curiosidad, ser críticos o merecer calificación alta.

Por último, Qingrong y Liming (2019) estudian la construcción de las identidades autoral, discursiva y autobiográfica (Burgess e Ivanič, 2010; Ivanič, 1998) de estudiantes chinos, al observar las prácticas de citación en tres versiones de tesis de maestría en el área de lingüística. En tres borradores y dos entrevistas por participante, los autores analizan idiomas, tipo y uso de documentos referenciados, y revelan las negociaciones en las construcciones de identidades académicas de los estudiantes, a raíz de las preferencias por ciertas publicaciones, así como las decisiones y creencias que los relacionan con su identidad como escritores todavía noveles en la comunidad académica. Sin embargo, no adoptan un enfoque longitudinal para los análisis, de manera que no se relevan en las posibles modificaciones que pudieran haber tenido lugar durante ese tiempo de escritura.

Los resultados de tales trabajos constatan el potencial de estos enfoques para visibilizar la complejidad detrás de las elecciones de citación, permeadas por una identidad en construcción. No obstante, a nivel de posgrado, aún está pendiente adelantar estudios que permitan relevar más explícitamente las decisiones de compromiso, participación, concesión o incluso resistencia respecto a los discursos privilegiados de determinadas comunidades, pese a que investigaciones de este carácter resultan relevantes para comprender la agencia y las experiencias de los escritores. Asimismo, aunque la construcción de la identidad académica ocurre de forma paulatina y procesual, el diseño longitudinal ha sido escasamente explorado para informar sobre las tensiones y transformaciones identitarias, pese a que la identidad constituye una experiencia vivida y negociada conforme se participa de una comunidad (Wenger, 2001), de manera que demanda un estudio temporal más sostenido que permitan captar modificaciones y negociaciones.

Desde una perspectiva múltiple, social y discursivamente construida de la identidad (Gee, 2000), este artículo indaga cómo la construcción de la identidad académica de doctorandos en Educación se relaciona con las formas de citar, analizadas en distintas versiones de un mismo escrito e integradas con sus testimonios. La pregunta que guía este estudio es: ¿de qué manera las decisiones de citación durante la escritura de la introducción de una revisión de literatura se vinculan con los procesos de construcción de identidad de los estudiantes doctorales? Para responderla, se construyeron dos preguntas específicas: ¿cómo se distribuyen y varían longitudinalmente los propósitos de citación usados por los estudiantes? ¿Qué razones atribuyen los participantes a estas decisiones de uso?

\section{Marco teórico}

Este trabajo se inscribe en el marco de las literacidades académicas y utiliza, particularmente, la concepción sobre identidad propuesta por Ivanič (1998). Esta corriente considera la literacidad como una práctica social relacionada con todas las formas de emplear el lenguaje escrito de manera situada, en un marco de usos dominantes 
o prototípicos (Gee, 2008). En este panorama, la identidad se concibe como un constructo social y, por ende, como un fenómeno inestable e indeterminado, en el que las posibilidades para el "yo" son activas, dinámicas y están abiertas a la contestación y al cambio (Ivanič, 1998; Lillis, 2013).

En su participación dentro de una comunidad, el sujeto establece vínculos con otros miembros, se identifica, se compromete, acepta, resiste o ayuda a modificar las prácticas y creencias sobre la escritura que predominan dentro de ese sistema ideológico (Ivanič, 1998; Zavala, 2011). De esta manera, según las circunstancias sociales y las prácticas letradas a las que ha tenido acceso y con las que se ha alineado, cada persona va construyendo su repertorio de elecciones discursivas, para responder a situaciones de comunicación (Ivanič, 1998; Lillis, 2013). Consecuentemente, participar de un nuevo contexto cultural, como iniciar un ciclo de formación educativa, constituye una nueva fase de desarrollo de la literacidad (Ivanič, 1998). Esta participación en prácticas nuevas o menos familiares involucra una ampliación negociada del repertorio comunicativo personal, así como posibles tensiones entre las previas y nuevas creencias sobre la literacidad (Lillis, 2013). Todo ello repercutirá en sus decisiones de escritura.

En cada acto escrito interactúan y se tensionan diferentes dimensiones de la identidad del escritor. Ivanič (1998) propone tres formas interrelacionadas de pensar esta identidad: en primer lugar, se encuentra el "yo autobiográfico", que consiste en que el bagaje social y discursivo permea la escritura. Aquel está en constante cambio, como el desarrollo de su propia vida, y abarca las creencias, los valores e intereses del escritor, pero también prácticas, hábitos y preferencias de escritura (Burgess e Ivanič, 2010).

En segundo lugar está el "yo discursivo", traducido en la imagen que el escritor genera de sí en el texto, consciente o inconscientemente. Esta construcción, basada en las elecciones lingüísticas y discursivas, se negocia en el contexto social de la escritura y está influenciada por los valores, las creencias y las relaciones de poder que rodean al escritor.

Por último, el "yo autoral" habla de cómo el escritor se posiciona y se afirma; cuánta autoridad reclama, se atribuye e imprime en el texto (Burgess e Ivanič, 2010; Ivanič, 1998). Pese a su íntima relación con el "yo discursivo", el "yo autoral" refiere específicamente al aspecto interpersonal de la identidad; es producto de la autopercepción como escritor y del nivel de responsabilidad que se desea asumir. Se construye mediante usos del lenguaje que no están vinculados a discursos específicos, sino a usos particulares (Burgess e Ivanič, 2010).

Los yoes autobiográfico, discursivo y autoral, construidos en cada acto escrito y atribuidos a un escritor real y particular, están permeados por un cuarto aspecto: las posibilidades sociales de ser - prototípicas y variables en el tiempo-, disponibles en determinado contexto (ser un tipo de investigador, estudiante, profesor, entre otras), que son más o menos valoradas en este (Burgess e Ivanič, 2010; Ivanič, 1998).

\section{Método}

Se plantea un diseño longitudinal de cuatro casos con alcance exploratorio. ${ }^{1}$ En concordancia con una mirada sociocultural de la escritura, se opta por un acercamiento "ecológico" (Barton y Hamilton, 2004; Ivanič, 1998), que permita una integración entre literacidad y contexto. Para esto, se realizó un análisis discursivo interpretativo de las introducciones de varias versiones de las revisiones sistemáticas de la literatura (RSL) de los participantes y se integró con un análisis de contenido de entrevistas basadas en textos.

\section{Contexto del estudio}

La universidad de estudio es chilena, altamente selectiva y listada en los primeros 10 lugares del ranking

1 Todos los procedimientos de recolección de datos fueron revisados y aprobados por el Comité de Ética de la institución estudiada. Así mismo, todos los participantes firmaron consentimientos informados previo inicio del estudio. 
de investigación de Scimago (2020). Su programa doctoral en Educación está acreditado por la Comisión Nacional de Acreditación de Chile y funciona desde hace tres décadas. Durante el primer año y medio se cursa la secuencia de seminarios de investigación: tres cursos destinados a la elaboración de una RSL (Feak y Swales, 2009) sobre el posible tema de investigación. El resultado esperado al finalizar esta secuencia es un artículo académico publicable.

Esta estructura permite un estudio sostenido de familiarización con una práctica letrada que es elaborada, iterada y retroalimentada durante un año y medio, además de que se sitúa al inicio del proceso de formación y repercute en el desempeño futuro del investigador. La revisión de literatura es una tarea fundamental en la formación doctoral (Feak y Swales, 2009), que demuestra la capacidad del estudiante para evaluar información académica, sintetizar la evidencia en el campo y construir una base crítica para la investigación (Boote y Beile, 2005). Es el momento en que el doctorando se compromete con perspectivas teóricas y encuentra un lugar, entre la comunidad, desde el cual dialogar y posicionarse como investigador (Wisker, 2015), como inicio del proceso de construcción de esta identidad académica.

Durante el segundo y tercer seminario, los estudiantes reciben instrucción explícita en escritura, incluyendo conceptos como recursos metadiscursivos (Hyland, 2005) y movidas retóricas (Swales, 1990) - establecer el territorio, establecer el nicho y ocupar el nicho- para orientar la estructuración de introducciones. Este modelo sobre cómo se disputa la credibilidad y la legitimidad en el campo disciplinar es particularmente relevante para este estudio, dado que su análisis está focalizado en el apartado introductorio. Se ha escogido trabajar con introducciones, ya que en estas, frecuentemente, se despliegan esfuerzos retóricos por adherirse a un determinado campo de estudios y, a su vez, revisar y discutir literatura existente para establecer un vacío de investigación (Swales, 1990; 2004).

\section{Muestra}

Se utilizó una muestra intencionada de cuatro doctorandos ingresantes en el mismo año al programa en Educación de la universidad de estudio, quienes seleccionaron sus seudónimos y cuyas edades oscilaban entre los 28 y los 34 años al comenzar la investigación. Dos son hombres: Pierre, licenciado en Ciencias Exactas, y Andy, físico. Dos son mujeres: Sara, bióloga y ecóloga, y Pepita, economista.

\section{Recolección de datos}

Al finalizar cada seminario, se les solicitó a los estudiantes la versión entregada de su RSL. Así, se contó con un total de 12 textos, 3 versiones por escritor, para el análisis de los propósitos de citación. Tras este análisis, a partir del cual se levantaron gráficas de distribución, se convocó a cada participante a una entrevista semiestructurada basada en textos (Ivanič, 1998), cuya pauta fue previamente sometida a juicio de un experto en el área y ajustada según sus recomendaciones.

En estas entrevistas, se les explicó a los estudiantes el análisis textual realizado y se les mostró la distribución de propósitos captada en cada una de sus versiones, como base para dialogar y reflexionar acerca de estos usos en el año y medio que duró su escritura. La instrucción explícita sobre escritura recibida por ellos dentro de los seminarios habilitó el uso de cierto metalenguaje propio de la escritura durante los encuentros. A partir de las gráficas de distribución, se les instó a opinar y expresarse sobre los resultados, así como su coincidencia con la idea que tenían sobre sus decisiones. Posteriormente, se indagó sobre los propósitos más y menos utilizados, y las variaciones entre versiones, de existir. Las entrevistas fueron transcritas en Word, con ortografía estándar.

\section{Análisis de datos}

Acorde con una concepción dinámica y situada de la escritura, se optó por emplear categorías emergentes, descartando el uso de solo una taxonomía o 
clasificación predeterminada que limitara la riqueza de los resultados y desestimara los contextos en que ocurren las prácticas.

Además, se utilizaron herramientas de análisis del discurso del tipo interpretativo para levantar estas categorías, con el fin de poder clasificar y captar la progresión de incorporación de las citas de las introducciones de las tres versiones de cada participante.

Para ello, se revisaron previamente otras propuestas de clasificación de funciones retóricas o motivos de citación que la literatura ha planteado para analizar documentos académicos de expertos o investigadores en formación. Entre estos antecedentes están:

1. El modelo de funciones discursivas para consenso y disenso levantado por Meza y Sabaj (2016), a partir de 36 tesis de lingüística de pregrado, magíster y doctorado.

2. La propuesta de Petrić (2007) para estudios retórico-contrastivos de tesis de maestría de

Tabla 1 Propósitos de citación levantados

\begin{tabular}{|c|c|c|}
\hline Propósito & Operacionalización & Ejemplo \\
\hline $\begin{array}{l}\text { Identificar brechas } \\
\text { (BRE) }\end{array}$ & $\begin{array}{l}\text { La cita sustenta vacíos o brechas en el conocimiento, } \\
\text { susceptibles de ser investigadas. A diferencia de la } \\
\text { categoría opo, aquí coinciden escritor y autor citado. }\end{array}$ & $\begin{array}{l}\text { "Se sabe poco de las características del estudiante y su entorno } \\
\text { familiar y escolar relacionados con abandono en educación } \\
\text { secundaria en estudiantes con discapacidades (Doren et al., 2014)" } \\
\text { [Pepita3].* }\end{array}$ \\
\hline $\begin{array}{l}\text { Señalar importancia } \\
\text { del territorio (TERR) }\end{array}$ & $\begin{array}{l}\text { La cita ayuda a relevar la importancia del tema en el } \\
\text { territorio —-término acuñado por Swales (1990)— } 0 \\
\text { campo científico del estudio. }\end{array}$ & $\begin{array}{l}\text { "Los docentes son los actores centrales en las reformas educacionales } \\
\text { que los gobiernos buscan implementar a nivel de escuela (Garet et } \\
\text { al., 2001; Porter et al., 2003)" [Pierre3]. }\end{array}$ \\
\hline $\begin{array}{l}\text { Señalar evoluciones } 0 \\
\text { tradiciones de estudio } \\
\text { (TRAD) }\end{array}$ & $\begin{array}{l}\text { La cita se usa para explicar evoluciones o tradiciones } \\
\text { de un término o campo. Indica cómo ha sido abordado } \\
\text { un campo en el tiempo o los cambios generados. }\end{array}$ & $\begin{array}{l}\text { "Una de las estrategias de enseñanza distintivas que se ha } \\
\text { promovido desde hace décadas en el campo de la educación de } \\
\text { la física lo conforman los laboratorios de física (Hanif et al., 2009; } \\
\text { Kirkup et al., 1998)" [Andy2]. }\end{array}$ \\
\hline $\begin{array}{l}\text { Introducir relevancia } \\
\text { sobre el nicho (Nㄷㅇ) }\end{array}$ & $\begin{array}{l}\text { La cita ayuda a relevar la importancia del objeto } \\
\text { de estudio, correspondiente al nicho —-érmino } \\
\text { acuñado por Swales (1990)—, es decir, la } \\
\text { profundización de un aspecto particular del campo } \\
\text { científico. Se relaciona con afirmaciones subjetivas de } \\
\text { sustentación. }\end{array}$ & $\begin{array}{l}\text { "Los programas de DPD [desarrollo profesional docente] aparecen } \\
\text { como una solución para la mayoría de los problemas que se producen } \\
\text { dentro de las escuelas cuando se implementan nuevas reformas } \\
\text { curriculares que afectan directamente a las aulas, como por ejemplo } \\
\text { los cambios del } 2012 \text { a las bases curriculares de matemática en } \\
\text { educación básica (Ministerio de Educación, 2012)" [Pierre3]. }\end{array}$ \\
\hline Definir conceptos (DEF) & $\begin{array}{l}\text { La cita apoya definiciones u operacionalizaciones } \\
\text { de términos o conceptos. Puede clasificar o describir } \\
\text { características o elementos que componen un } \\
\text { concepto. También puede aludir a la diversidad de } \\
\text { concepciones o aplicaciones de un concepto. }\end{array}$ & $\begin{array}{l}\text { "La palabra estética proviene del griego aisthesis, que significa sentir } \\
\text { y se relaciona con sensibilidad (Ghisloti, et al., 2016)" [Saral]. }\end{array}$ \\
\hline $\begin{array}{l}\text { Incorporar evidencia } \\
\text { (EVI) }\end{array}$ & $\begin{array}{l}\text { La cita sirve para hacer reporte expreso de } \\
\text { resultados y evidencia empírica sobre el tema de } \\
\text { estudio. Responde a qué se conoce o se sabe del } \\
\text { campo. }\end{array}$ & $\begin{array}{l}\text { "Las investigaciones exponen que los estudiantes con discapacidades } \\
\text { de aprendizaje muestran mayores tasas de abandono que sus pares } \\
\text { sin dichas discapacidades (Cobb et al., 2006; Doren et al., 2014)" } \\
\text { [Pepita3]. }\end{array}$ \\
\hline $\begin{array}{l}\text { Introducir una } \\
\text { oposición (OPO) }\end{array}$ & $\begin{array}{l}\text { Se presenta una cita para criticarla. A diferencia de } \\
\text { BRE, aquí el escritor se opone a la idea citada. }\end{array}$ & $\begin{array}{l}\text { "Los logros de aprendizaje entregados por la AAPT [American } \\
\text { Association of Physics Teachers] (Kozminski et al., 2014) solamente } \\
\text { representan recomendaciones y, por tanto, su alineación con el } \\
\text { currículo estaría sujeta a discusión" [Andy3]. }\end{array}$ \\
\hline
\end{tabular}

* Convención: autor, número de versión. 
estudiantes cuya primera lengua no es el inglés, que formula funciones de atribución, ejemplificación, mención a referencias adicionales, declaración de uso, aplicación, evaluación, enlaces entre fuentes y comparación. Esta propuesta fue ajustada por Sánchez-Jiménez (2013; 2016) para analizar memorias de máster escritas en español por estudiantes filipinos y españoles.

3. La tipología de 15 funciones de Weinstock (1971), utilizada después por Sabaj y Páez (2011) para comparar artículos entre disciplinas.

4. La taxonomía de motivos para citar, basada en entrevistas, propuesta por Erikson y Erlandson (2014), que incluye cuatro categorías - argumentación, alineamiento social, alineamiento comercial y datos- y sus correspondientes subdivisiones. Finalmente, el marco conceptual de Swales (1990) sirvió como herramienta analítica e informó la creación de algunos propósitos de citación.

A partir de estas propuestas, se analizaron cuatro versiones de las introducciones de la RSL (30 \% del corpus) para levantar las categorías especificadas en la Tabla 1.

Cada introducción se dividió en cláusulas citadas, que fueron clasificadas según el(los) propósito(s) correspondiente(s). En este procedimiento, se

Tabla 2 Distribución de citas en la introducción de la RSL en tres momentos

\begin{tabular}{lcccccc}
\hline Autor & \multicolumn{2}{c}{ RSL1 } & \multicolumn{2}{c}{ RSL2 } & \multicolumn{2}{c}{ RSL3 } \\
\cline { 2 - 7 } & Citas & $\begin{array}{c}\text { Total } \\
\text { palabras }\end{array}$ & Citas & $\begin{array}{c}\text { Total } \\
\text { palabras }\end{array}$ & Citas & $\begin{array}{c}\text { Total } \\
\text { palabras }\end{array}$ \\
\hline Sara & 23 & 1362 & 22 & 1192 & 16 & 1023 \\
Andy & 18 & 1034 & 20 & 998 & 23 & 1445 \\
Pepita & 20 & 933 & 19 & 934 & 19 & 1052 \\
Pierre & 19 & 1033 & 37 & 1894 & 39 & 1808 \\
\hline
\end{tabular}

RSL: Revisiones sistemáticas de la literatura. analizó un total de 275 citas, distribuidas como indica la Tabla 2.

Para asegurar la confiabilidad de la codificación, las investigadoras revisaron, refinaron y calibraron las categorías. Además, se doblecodificó el $25 \%$ del corpus y se obtuvo un Kappa de Cohen de $k=0,89$, considerado un acuerdo óptimo (Fleiss et al., 2003). Luego, se normalizaron las recurrencias, dividiendo el número de veces que un propósito era utilizado en la introducción de una versión por el total de citas incluidas en esta.

Por último, dado que una misma referencia puede cumplir más de un motivo o función (Erikson y Erlandson, 2014; Hyland, 2004), si una cita era clasificada en dos propósitos, se contabilizaba en cada categoría como 0,5 , lo que sucedió en una sola ocasión.

\section{Resultados y discusión}

A continuación se presenta lo hallado según las preguntas específicas planteadas. En este sentido, primero se muestran y comentan las gráficas de distribución del análisis textual, que permiten visualizar de manera longitudinal la variabilidad de los propósitos de citación entre las diferentes versiones de cada participante. Posteriormente, se analizan los resultados emergidos de las entrevistas.

\section{Propósitos de citación: una mirada longitudinal}

Para atender a la primera pregunta específica sobre cómo se distribuyen y varían longitudinalmente los propósitos de citación usados por los estudiantes, se realizó un análisis de frecuencias, cuyos resultados se muestran en la Tabla 3, logrados tras la clasificación de citas y la normalización de recurrencias.

Lo primero que destaca son las preferencias de cada participante al momento de seleccionar los propósitos de citación para construir su introducción. Pierre muestra predilecciones por funciones como EVI, TRAD o NICHO, mientras que Pepita y Andy tienden, en susúltimas dosversiones, a usar más 
Tabla 3 Distribución de propósitos de citación en las introducciones de RSL en tres momentos

\begin{tabular}{|c|c|c|c|c|c|c|c|}
\hline \multirow{2}{*}{$\begin{array}{l}\text { Versión de } \\
\text { RSL según } \\
\text { autor }\end{array}$} & \multicolumn{7}{|c|}{ Propósitos de citación } \\
\hline & $\begin{array}{l}\text { BRE } \\
\text { (\%) }\end{array}$ & $\begin{array}{l}\text { TERR } \\
(\%)\end{array}$ & $\begin{array}{l}\text { TRAD } \\
(\%)\end{array}$ & $\begin{array}{l}\text { NICHO } \\
\text { (\%) }\end{array}$ & $\begin{array}{l}\text { DEF } \\
(\%)\end{array}$ & $\begin{array}{l}\text { EVI } \\
\text { (\%) }\end{array}$ & $\begin{array}{l}\text { OPO } \\
\text { (\%) }\end{array}$ \\
\hline Saral & 4 & 9 & 4 & 30 & 52 & 0 & 0 \\
\hline Sara2 & 9 & 9 & 11 & 9 & 55 & 7 & 0 \\
\hline Sara3 & 13 & 13 & 9 & 13 & 50 & 3 & 0 \\
\hline Andyl & 6 & 6 & 50 & 6 & 33 & 0 & 0 \\
\hline Andy2 & 10 & 0 & 15 & 25 & 15 & 35 & 0 \\
\hline Andy3 & 9 & 0 & 9 & 30 & 17 & 30 & 4 \\
\hline Pepital & 10 & 30 & 0 & 10 & 0 & 50 & 0 \\
\hline Pepita2 & 11 & 32 & 0 & 5 & 0 & 53 & 0 \\
\hline Pepita3 & 11 & 32 & 0 & 5 & 0 & 53 & 0 \\
\hline Pierrel & 0 & 0 & 16 & 58 & 26 & 0 & 0 \\
\hline Pierre2 & 14 & 8 & 32 & 32 & 5 & 8 & 0 \\
\hline Pierre3 & 13 & 8 & 41 & 26 & 5 & 8 & 0 \\
\hline
\end{tabular}

RSL: Revisiones sistemáticas de la literatura; BRE: Identificar brechas; TERR: Señalar importancia del territorio; TRAD: Señalar evoluciones o tradiciones de estudio; NICHO: Introducir relevancia sobre el nicho; DEF: Definir conceptos; EVI: Incorporar evidencia; OPO: Introducir una oposición.

citas para incorporar evidencia (EVI). Asimismo, Pepita presenta mayores usos de TERR, categoría que es muy poco utilizada por Andy. Entre tanto, Sara muestra preferencia por DEF.

Ninguno de los participantes, excepto Andy en su última versión, hace alusión a un autor para introducir una oposición (OPO). Este último hallazgo coincide con los de Meza y Sabaj (2016), respecto al mínimo uso de fuentes para funciones discursivas de disenso. Este comportamiento podría vincularse a la dificultad que supone, para los doctorandos, construir un yo autoral, una voz que se distancia o tensiona las ideas o hallazgos de otros autores.

Por otro lado, desde una perspectiva longitudinal intraestudiante, que permite apreciar el proceso de cada escritor, destaca la poca variabilidad de Pepita y Sara en cuanto a los propósitos para citar. En contraste con esta poca variabilidad, la primera versión de Andy se diferencia de las otras dos, pues, en un principio, muestra mayor tendencia a forjar su introducción a partir de DEF O TRAD, sin incorporar evidencia del campo (EVI). Al contrario, en las últimas dos versiones expone una predominancia por NICHO y Evi. Cabe explicitar que Andy cambió el tema de su revisión entre la primera y la segunda versión, lo que podría explicar en parte la modificación de sus patrones.

Así como Andy, Pierre muestra una variabilidad entre la primera versión y las dos últimas, aunque él mantuvo su tema de revisión, como Sara y Pepita. En detalle, la primera versión de Pierre muestra el uso de solo tres propósitos de cita, mientras que las demás versiones incorporan seis. Además, en la primera versión, más de la mitad de las citas se usan para NICHO, propósito que es utilizado también en las demás versiones, pero en menor cantidad que TRAD.

Finalmente, llama la atención que todos los participantes, excepto Pepita, muestran longitudinalmente un cambio en el modo de citar entre la primera y la segunda versión, que es menor a las diferencias encontradas entre la segunda y la tercera.

\section{Decisiones de uso de propósitos}

Respecto a la segunda pregunta específica acerca de las razones que atribuyen los participantes a estas decisiones de uso, sus reflexiones sobre los resultados mostraron que, en esta práctica, la construcción de su identidad como escritores está moldeada por una negociación entre las identidades que traen consigo al acto de escritura y la imagen que pretenden proyectar y que se encuentran construyendo.

\section{Identidades previas: estilo e identificaciones disciplinarias}

Cuando se les insta a hablar sobre los propósitos por los que parecen haber tenido mayor y menor preferencia, los participantes aluden a usos que se han vuelto hábitos con los que se sienten cómodos, que relacionan con lo que consideran "su estilo" de escritura, y que muchas veces son una respuesta a 
sus posturas epistemológicas y filiaciones disciplinares. Un caso ilustrativo es la respuesta de Sara sobre su predilección por DEF:

Creo que es muy de la biología, de mi formación inicial. Uno define mucho, explica bien el concepto. Para mí siempre es muy importante [decir:] "yo estoy hablando de esto y lo entiendo de esta manera”. Sí, creo que yo soy muy así, de entender los conceptos bien.

Sara declara identificación y apropiación con su disciplina de origen y alineación con las creencias de esta al presentar el recurso como una convención de la comunidad, y a la vez incorporar una explicación personal sobre el valor de este uso: "soy muy asi".

De manera similar, cuando Pepita observa la distribución de propósitos de citación de sus textos, señala EVI y exclama "me parece muy yo". Luego añade:

Quizá es una costumbre. Una cuestión medio matemática, pero hasta que uno no ve... como dicen, "ya, pero muéstrame las cifras”. Hasta que uno no ve las cifras, no sabe la gravedad del problema. Porque todo el mundo te puede decir: "en verdad tenemos que hacer que los niños tengan más acceso a la educación”. Ya, pero y ¿de qué acceso estamos hablando?, ¿es un 10 contra un 11 o es un 20 contra un 40 ? Eso me lo da la evidencia.

Quedan claros los fundamentos epistemológicos que inspiran a Pepita a priorizar este propósito de citación; para ella, la evidencia numérica, propia del campo de la economía, es la manera de constatar la existencia de un problema. Tal identificación con un tipo de persona, cuya incorporación se ha vuelto "costumbre", es reforzada al hablar sobre los propósitos menos utilizados:

Pepita: Esto es muy mío también, no señalar definiciones o tradiciones del estudio.

Entrevistadora: ¿Qué opinas de eso?

Pepita: Me parece muy cuali [risas].

Pepita atribuye su elección al establecimiento de una dicotomía entre convenciones "cuantitativas/cualitativas", en la que se identifica claramente como cuantitativa. Este ejemplo expone, además, cómo estas identidades que se traen consigo al acto de escritura están representadas por decisiones de incorporación de ciertos recursos que comulgan con creencias propias de las comunidades objeto de identificación, pero también por la abstinencia o resistencia al uso de otros menos valorados, como sería para "los cuantitativos", es decir, utilizar muchas citas sobre definición (DEF) o tradición (TRAD) durante la introducción.

Otro caso que ejemplifica esta resistencia es cuando Andy explica por qué prefiere no usar citas para señalar importancia del territorio (TERR) en sus escritos:

\begin{abstract}
A mí eso no se me da, la verdad. Cada vez que me tengo que enfrentar en escrituras de esa línea, no me siento cómodo, no me siento parte de esa línea o de esa función, y siento que no soy yo. De hecho, la primera introducción que escribí fue muy en esa línea de señalar la importancia del territorio, pero no me sentía cómodo; escribí dos párrafos y los eliminé, porque fue tratar de copiar un poco lo que habían hecho otros autores y no sentía que fuera yo el que estaba haciendo eso. Entonces, si no estoy cómodo, no lo hago, y no lo hice [casi] en ninguna de las tres [versiones]. Es por eso, porque para mí es más, no sé si de los practitioner [profesionales], pero más de esta gente... como con estos policy briefs [informes de política], yo lo veo, van en esa línea y no me agrada. Ese estilo a mí no se me da. Si pudiera evitarlo a futuro, lo evitaría a toda costa.
\end{abstract}

Este fragmento ilustra dos puntos: 1) insiste en el vínculo que los participantes tienden entre el "tipo de persona” (Gee, 2000) con que se identifican y el uso de determinados recursos comunicativos; Andy no "se siente cómodo", "no es su estilo" usar determinado propósito, porque lo vincula con una imagen de la que quiere distanciarse. Y 2) esta resistencia contrasta con el recuerdo de cómo intentó ceder a este recurso discursivo, pero su sentimiento de inadecuación fue mayor. Pese a haber tenido instrucción en el concepto de las movidas retóricas de Swales y saber que es un uso extendido y valorado por la comunidad académica, Andy se mantiene fiel a sus creencias sobre la escritura, incluidas por su yo autobiográfico y al tipo de persona con que se identifica como escritor académico.

Los ejemplos expuestos hablan de creencias, filiaciones y patrones de elección que los estudiantes 
han construido previamente y que evalúan e incorporan a este acto de escritura. Así, en estos tres casos, se advierte cierta tensión entre los modos de construir conocimiento que privilegian y otras formas valoradas en su nuevo contexto de aprendizaje y en el campo de la educación.

Pero de los testimonios y de la captación de la variabilidad longitudinal entre las versiones sobresalieron también reflexiones sobre la ampliación de los conjuntos de recursos discursivos personales, durante este periodo, producto de la participación en nuevas prácticas. Muestra de ello es la respuesta de Pierre sobre el aumento de tipos de propósitos usados entre su primer y segundo borrador: "Absolutamente. En el primero todavía me hacían falta algunas cosas que fui desarrollando, descubriendo la importancia de la cita para [énfasis] otras cosas". En retrospectiva, él interpreta esta variación como una limitación o "carencia" inicial de recursos, posteriormente descubiertose incorporados.

Según los testimonios, las decisiones de selección de ciertos propósitos de citación están orientados por las identidades previas, que se traducen en puntos de referencia propios y en alineaciones con determinadas tradiciones, que pretenden conservar ciertos valores e identificaciones disciplinares. En sus explicaciones, puede entenderse la manera en que los estudiantes se apropian de su discurso y se sienten fielmente reflejados en sus elecciones discursivas. Términos como "comodidad", "costumbre" o "estilo propio", y expresiones como "muy yo", "no se me da" o "yo soy asi”, apelan al yo autobiográfico, sus filiaciones con comunidades y tipos de personas con que se identifican; pero también sugieren un yo autoral construido mediante usos particulares, que denominan "estilo", erigido desde su participación en comunidades que privilegian otras prácticas, que se tensionan con las privilegiadas en su nuevo contexto y con las posibilidades de ser en el campo de la educación.

Además, entendiendo el estado inestable y de constante construcción de la identidad en esta etapa formativa, las comparaciones longitudinales entre versiones permiten relevar evidencias de ampliación de estos repertorios comunicativos, que componen la identidad del escritor, eventos esperables en esta primera fase de formación doctoral, que involucra la familiarización con prácticas nuevas o poco conocidas.

\section{Imagen en construcción y a proyectar}

Otras elecciones discursivas parecen orientarse, conscientemente o no, a proyectar determinada imagen al lector y a la comunidad de destino. Esta imagen dialoga con la forma en que los participantes se van construyendo como investigadores. Esta manifestación se cristaliza, primero, en la intención de escribir estratégicamente para cumplir las expectativas del lector y ser considerado parte de la comunidad a la que se busca pertenecer; y, segundo, en la forma desde la cual buscan posicionarse en su campo. Este resultado, que fluctúa entre la construcción del yo discursivo y autoral de los entrevistados, se condice con lo hallado en el estudio de Harwood y Petrić (2012), que explora la idea de "actuación" de Goffman, y encuentra que, al momento de decidir qué citar, las participantes buscan anticiparse a las posibles expectativas y formas de influenciar a su lector.

Acerca de lo primero, las decisiones de uso u omisión de determinados propósitos responden al cumplimiento de las expectativas de sus lectores y tienden un vínculo entre los participantes y su forma de posicionarse. Esto resultó común al conversar sobre opo, ausente en todos los textos, a excepción de la tercera versión de Andy. Frente a esta elección discursiva, él señala:

[...] sería raro no introducir una oposición a un nivel de doctorado. Uno esperaría, de hecho, me hace sentido, que se pida una oposición clara en la introducción, y eso igual a uno le da una propiedad como autor; y me parece ideal, me parece estupendo que al menos se vea plasmada una oposición en mis textos.

En esta evaluación de las posibilidades de ser en el contexto de la formación doctoral, Andy muestra poseer una imagen definida de las expectativas que se tienen de una persona "a nivel de doctorado" y 
que atañen a la capacidad de sentar abiertamente una posición propia en contra de la postulada por otro autor, lo que requiere asumir mayor nivel de autoridad y presentar un yo discursivo contundente. Por ende, se enorgullece de haber actuado "a la altura” en su última versión; haberse proyectado como escritor crítico (yo discursivo) y con autoridad, propio de un doctorando.

Algo semejante puede interpretarse en Pierre, quien enfatiza en la forma como realiza una crítica que releva su voz, pese a estar sustentada con otra referencia:

[...] quizás digo "al igual que Lindvall” y creo que sí me apoyo en otro autor, pero aun así critico al otro autor. No solo, pero hay una voz propia. Entonces, menciono a un autor, menciono otro que está en contra y yo digo: "yo tengo la misma posición que este frente a esto y que agregaría estos otros dos puntos". [...] De hecho, creo que es bastante bueno, porque te posiciona frente al lector y te dice para dónde va a ir el estudio.

Pierre insiste en haberse posicionado autoralmente frente al lector mediante una crítica, y que su voz no ha sido opacada por respaldarse en otro autor. Al parecer, él también busca construir un yo discursivo que cumpla con esa expectativa del lector de encontrar un autor crítico y posicionado. Ambos estudiantes muestran preocupación por alcanzar un comportamiento que consideran altamente valorado en la academia.

Por su parte, frente a esta práctica, Pepita asume una estrategia distinta:

Esto [оpo] nunca lo haría en una introducción, porque me parece que es quitarme puntos de inmediato. Imagínate que me está revisando una persona a la que yo critico, ¡no me lee más, nunca en la vida del trabajo para adelante! [risas].

A diferencia de Pierre y Andy, quienes consideran que en la formación doctoral una posibilidad de ser altamente valorada es mostrarse como escritores con autoridad en la introducción, Pepita prefiere silenciar esta posición y presentarla en otros momentos del texto. Ella considera esto una mejor estrategia para lograr cierta membresía o aceptación por parte de la comunidad lectora. En todos, no obstante, la decisión de incorporar o no el recurso está dictada por forjar aquella identidad que será más valorada en el contexto y que desean proyectar en el lector.

Respecto a lo segundo, fue también común que los participantes justificaran algunas decisiones discursivas como "necesarias", dado su objeto de investigación. Un ejemplo es cuando Sara explica por qué incorpora poca evidencia (EVI):

\section{[...] estoy trabajando harto con filósofos, donde hemos estado trabajando mucho en hacer una contribución ojalá teórica a un campo. [...] también el campo que estoy abordando va más hacia cómo están nuestras es- tructuras de pensamiento, nuestros patrones; por eso quiero más cosas teóricas.}

Sara les adjudica este comportamiento a sus prioridades en términos de la contribución que pretende realizar. En efecto, estas decisiones, lejos de ser taxativas, están supeditadas a elecciones de posicionamiento dentro del campo del saber y frente a su objeto de estudio. Mediante esta priorización de propósitos, ella se está construyendo como cierto tipo de investigadora dentro del campo, y el uso del plural "hemos" sugiere su identificación o membresía deseada del grupo con el que trabaja y al que busca dirigirse, en el ámbito de la filosofía de la educación.

Andy, por su parte, aclara que la variabilidad en el uso de propósitos entre sus versiones se debe parcialmente al cambio de tópico entre la primera y la segunda versión. Manifiesta que la novedad del primer tema requería de determinados usos: "yo la armé [la introducción] señalando las evoluciones, porque en ese contexto era importante dar cuenta de la evolución de ese campo", y más adelante añade que esta misma característica de "campo en construcción" le obligaba a efectuar mayores definiciones de conceptos (DEF), dado el poco consenso en el tema. Durante el segundo semestre, Andy investigó sobre los laboratorios de física, y en este, el propósito EVI cobró mayor 
relevancia: "esa introducción nace de esta falta de evidencia robusta en función del impacto que tienen los laboratorios".

Estas apreciaciones sobre la selección de propósitos según el objeto de investigación constituyen evidencia situada de la forma y el nivel de autoridad con que los participantes van asumiendo una posición en su campo y la construyen en el texto como cierto tipo de investigadores. Igualmente, ilustra cómo el yo discursivo se modifica parcialmente en las diferentes prácticas, conforme se va ampliando el repertorio de elecciones discursivas. De hecho, en cuanto a la disminución de TRAD, Andy recuerda:

[...] al final creo que estaba perdiendo el foco, dando cuenta de evoluciones y tradiciones. Ahí me acordé de esto de escribir más en simple. Y creo que la decisión fue eso: "no estoy haciendo un estado del arte de los laboratorios, quiero relevar un interés de estudio", y eso me dijo: "bueno, entonces, hay cosas que son innecesarias", y me pareció innecesario ahondar en las tradiciones de estudio de los labs [laboratorios], porque no era el foco. Por eso lo bajé, de hecho, casi a la mitad.

Él reconstruye una decisión consciente entre la segunda y la tercera versión sobre un cambio de foco en cómo presentar su investigación y, como consecuencia, posicionarse en su campo. Este concepto de cambio de foco es también utilizado por Pierre para interpretar variaciones entre propósitos en sus versiones:

Creo que mientras avanzaba el tiempo, fue cambiando el foco con el que fui planteando mi revisión de literatura. En un principio, solía escribir para que cualquier persona que leyera mi texto le encontrara sentido. Después fui quizá escribiendo más cercano a mi comunidad y dentro de eso traté de relevar las cosas que me parecieron más interesantes de la propia comunidad, y fijar bien con qué iba a trabajar y con quién, en lugar de, por ejemplo, definir muchos conceptos.

Pierre reconoce haber ido adecuando su escritura para su comunidad experta, lo que lo motivó a disminuir el uso de propósitos que relaciona con públicos más generales, como DEF, que podrían presentarlo como un investigador novel. Además, ambos testimonios permiten captar cambios dentro del periodo de escritura entre versiones, que sirven como insumo para reconocer que la producción de un texto involucra una constante negociación de elecciones para construir o proyectar un yo discursivo deseado.

En suma, algunas decisiones retóricas de los participantes están supeditadas a una anticipación de la reacción del lector objetivo, en una búsqueda por presentar un yo discursivo de investigadores pertenecientes a una comunidad y capaces de posicionarse frente a ella dentro de un campo de estudio, con voz propia, y más o menos crítica, según su yo autoral. Asimismo, las comparaciones longitudinales permiten reflexiones retrospectivas que ilustran procesos de ampliación del conjunto de recursos para actuar en situaciones comunicativas y la negociación entre decisiones de posicionamiento.

\section{Conclusiones}

Este estudio aporta a complejizar la relación entre las dimensiones que conforman la identidad del escritor y la manera como este se aproxima al uso de las citas en un acto escrito. Específicamente, los participantes incorporan su yo autobiográfico, relacionando sus preferencias por algunos propósitos de citación con conceptos como "comodidad", "hábito" o "estilo", que en ocasiones constituyen expresiones de comunión e identificación con determinadas posturas epistemológicas o disciplinarias, que implican una negociación con identidades previas de los participantes (Hoang y Pretorius, 2019). Asimismo, la autopercepción de los participantes como investigadores se encuentra en desarrollo. Esto repercute en cómo forjan sus yoes autoral y discursivo, y en cómo evalúan sus posibilidades de ser dentro de su contexto de formación.

El diseño longitudinal favoreció la captación de una nueva fase de desarrollo de la literacidad en los escritores, quienes amplían, en el tiempo, su repertorio comunicativo y modifican su forma de posicionarse ante la comunidad experta conforme participan de nuevas prácticas. Así, se insiste en 
la identidad como una experiencia vivida y negociada mediante la participación en comunidades (Wenger, 2001), y se asiste a la construcción de una identidad académica híbrida o múltiple en el acto escrito, donde las identidades previas se tensionan y negocian con otras nuevas o en proceso de construcción. Tales hallazgos ratifican la importancia de entender a los escritores como sujetos con agencia, que toman decisiones y reflexionan sobre su escritura (Zavala, 2011).

En virtud de lo anterior, haber integrado el análisis intertextual con los testimonios permitió comprender que la construcción de la identidad académica mediante la citación es compleja y no responde a intenciones únicas, sea de adherirse a ciertos usos privilegiados, reproducir determinados patrones sin cuestionarlos o resistirse a otros. Por esto, resulta importante continuar prestando atención a los testimonios respecto a las elecciones discursivas, en detrimento de generalizaciones o interpretaciones basadas en el texto como única fuente de investigación para informar la formación doctoral. Continuar realizando investigaciones sostenidas en el tiempo, y que vinculen diferentes fuentes de datos y procesos de análisis, permitirá ayudar a comprender la complejidad del proceso de escritura doctoral desde la perspectiva del escritor, valorar la agencia de los estudiantes y reconocer sus repercusiones y negociaciones identitarias.

Finalmente, cabe mencionar algunas limitaciones del presente estudio. Entre ellas, el uso de una muestra acotada y anidada a un contexto específico, que no permite la realización de generalizaciones, aunque sus hallazgos aportan a la discusión sobre la identidad de los investigadores en formación. Asimismo, el uso de entrevistas como método para comprender las intenciones de los escritores reconoce que no todas las elecciones de estos son conscientes (Ivanič, 1998); sin embargo, se considera que la reconstrucción y la reflexión que los participantes llevan a cabo son, en sí mismas, enriquecedoras.

\section{Agradecimientos}

A la Agencia Nacional de Investigación y Desarrollo (ANID) de Chile, folio 21180042, por la financiación para esta investigación.

\section{Referencias}

Ávila Reyes, N. (2018). Locales, regionales y cosmopolitas: análisis intertextual de artículos sobre escritura universitaria en América Latina Hispánica. En R. C. M. Pereira (Ed.), Escrita na universidade. Panoramas e desafios na América Latina. UFPB.

Barton, D. y Hamilton, M. (2004). La literacidad entendida como práctica social. En V. Zavala, M. Niño-Murcia y P. Ames (Eds.), Escritura y sociedad. Nuevas perspectivas teóricas y etnográficas (pp. 109-139). Red para el Desarrollo de las Ciencias Sociales en el Perú.

Boote, D. y Beile, P. (2005). Scholars before researchers: On the centrality of the dissertation literature review in research preparation. Education Researcher, 34(6), 3-15. https://doi.org/10.3102/0013189X034006003

Bornmann, L. y Daniel, H. (2008). What do citation counts measure? A review of studies on citing behavior. Journal of Documentation, 64(1), 45-80. https://doi. org/10.1108/00220410810844150

Burgess, A. e Ivanič, R. (2010). Writing and being written: Issues of identity across timescales. Written Communication, 27(2), 228-255. https://doi. org/10.1177/0741088310363447

Castelló, M., Iñesta, A. y Corcelles, M. (2013). Learning to write a research article: $\mathrm{Ph}$. D. students' transitions toward disciplinary writing regulation. Research in the Teaching of English, 47(4), 442-477. https:// www.jstor.org/stable/24397847

Castelló, M., McAlpine, L., Sala-Bubaré, A., Inouye, K. y Skakni, I. (2020). What perspectives underlie 'researcher identity'? A review of two decades of empirical studies. Higher Education, 81, 567-590. https://doi.org/10.1007/s10734-020-00557-8

Erikson, M. y Erlandson, P. (2014). A taxonomy of motives to cite. Social Studies of Science, 44(4), 625-637. https://doi.org/10.1177/0306312714522871

Fazel, I. y Shi, L. (2015). Citation behaviors of graduate students in grant proposal writing. Journal of English for Academic Purposes, 20, 203-214. https://doi. org/10.1016/j.jeap.2015.10.002 
Feak, C. y Swales, J. (2009). Telling a research story. Writing a literature review. University of Michigan Press. https://doi.org/10.3998/mpub.309338

Fleiss, J., Levin, B. y Cho Paik, M. (2003). Statistical methods for rates and proportions (3. ${ }^{\mathrm{a}} \mathrm{ed}$.). Wiley-Interscience. https://doi.org/10.1002/0471445428

Gee, J. (2000). Identity as an analytic lens for research in education. Review of Research in Education, 25, 99125. https://doi.org/10.2307/1167322

Gee, J. (2008). Social linguistics and literacies: Ideology in discourses (3. ${ }^{\mathrm{a}}$ ed.). Routledge. https://doi. org/10.4324/9780203944806

Giampapa, F. (2011). The politics of 'being and becoming' a researcher: Identity, power, and negotiating the field. Journal of Language, Identity and Education, 10(3), 132-144. https://doi.org/10.1080/1534845 8.2011 .585304

Goffman, E. (1959). The presentation of self in everyday life. Anchor Books.

Harris, R. (2005). Rhetoric and incommensurability. Parlor Press.

Harwood, N. y Petrić, B. (2012). "Performance in the citing behavior of two student writers". Written Communication, 29(1), 55-103. https://doi. org/10.1177/0741088311424133

Hoang, C. y Pretorius, L. (2019). Identity and agency as academics: Navigating academia as a doctoral student. In L. Pretorius, L. Macaulay y B. Cahusac de Caux (Eds.), Wellbeing in doctoral education. Insights and guidance from the student experience (pp. 143-151). Springer. https://doi.org/10.1007/978-981-13-9302-0_12

Hyland, K. (2004). Disciplinary discourses. Social interactions in academic writing. University of Michigan Press.

Hyland, K. (2005). Metadiscourse. Continuum.

Inouye, K. y McAlpine, L. (2019). Developing academic identity: A review of the literature on doctoral writing and feedback. International Journal of Doctoral Studies, 14, 1-31. https://doi.org/10.28945/4168

Ivanič, R. (1998). Writing and identity. The discoursal construction of identity in academic writing. Lancaster University. https://doi.org/10.1075/swll.5

Ivanič, R., Edwards, R., Barton, D., Martin-Jones, M., Fowler, Z., Hughes, B., ... Smith, J. (2009). Improving learning in college: Rethinking literacies across the Curriculum. Routledge. https://doi.org/10.4324/9780203881422

Leshem, S. (2020). Identity formations of doctoral students on the route to achieving their doctorate.
Issues in Educational Research, 30(1), 169-186. http://www.iier.org.au/iier30/leshem.pdf

Lillis, T. (2013). Sociolinguistics of writing. Edinburgh University Press. https://doi.org/10.1017/ cbo9781139164597.013

Meza, P. y Sabaj, O. (2016). Funciones discursivas de consenso y disenso en tesis de lingüística. Onomázein, 33(1), 385-411. https://doi.org/10.7764/ onomazein.33.23

Petrić, B. (2007). Rhetorical functions of citations in highand low-rated master's theses. Journal of English for Academic Purposes, 6(3), 238-253. https://doi. org/10.1016/j.jeap.2007.09.002

Qingrong, L. y Liming, D. (2019). Chinese MA student writers' identity construction in citation practices. Chinese Journal of Applied Linguistics, 42(3), 365385. https://doi.org/10.1515/CJAL-2019-0022

Sabaj, O. y Páez, D. (2011). Tipos y funciones de las citas en artículos de investigación de tres disciplinas. $\mathrm{Li}$ teratura y Lingüistica (22), 117-134. https://doi. org/10.4067/S0716-58112010000200009

Sánchez-Jiménez, D. (2013). Estudio transcultural de las funciones retóricas de las citas en el género académico de la memoria de máster. Argus-A Artes y Humanidades, 2(9), 1-59.

Sánchez-Jiménez, D. (2016). Implicaciones de la citación en la voz del autor en el discurso académico universitario: la memoria de máster escrita en español por estudiantes españoles y filipinos. Diálogo de La Lengua, VIII, 16-36. https://academicworks.cuny.edu/cgi/ viewcontent.cgi ?article $=1189 \&$ context $=$ ny_pubs

Sapienza, F. (2007). Ethos and research positionality in studies of virtual communities. In H. McKee y D. DeVoss (Eds.), Digital writing research. Techonologies, methodologies an ethical issues (pp. 89-106). Hampton Press.

Scimago. (2020). Scimago institutions rankings. Higher education. Chile- research rank. https://www.scimagoir.com/rankings.php? country $=$ CHL\&rankin $\mathrm{g}=$ Research\&sector=Higher\%20educ

Swales, J. (1990). Genre analysis: English in academic and research settings. Cambridge University Press.

Swales, J. (2004). Research genres. Exploration and applications. Cambridge University Press. https://doi. org/10.1017/CBO9781139524827

Tusting, K., McCulloch, S., Bhatt, I., Hamilton, M. y Barton, D. (2019). Academics writing. The dynamics of knowledge creation. Routledge. https://doi. org/10.4324/9780429197994 
Weinstock, M. (1971). Citation indexes. In A. Kent y H. Lancour (Eds.), Encyclopedia of library and information science, 5 (pp. 16-40). Marcel Dekker.

Wenger, E. (2001). Comunidades de práctica. Aprendizaje, significado e identidad. Paidós.

Wisker, G. (2015). Developing doctoral authors: Engaging with theoretical perspectives through the literature review. Innovations in Education and Teaching International, 52(1), 64-74. https://doi.org/10.1080/14 703297.2014 .981841

Zavala, V. (2011). La escritura académica y la agencia de los sujetos. Cuadernos Comillas, 1(1), 667-81. https:// www.fundacioncomillas.es/cuadernos-comillas/ cuadernos/el-espanol-escrito-en-contextos-contemporaneos/pdf/cuaderno.pdf

Cómo citar este artículo: Calle-Arango, L., Ávila Reyes, N. y Meneses, A. (2021). Construcción y transformaciones de las identidades académicas de estudiantes doctorales mediante la citación. Íkala, Revista de Lenguaje y Cultura, 26(2), 331-346. https://doi.org/10.17533/udea.ikala.v26n2a12 